Correspondence

Wouter J. Middelhoven

Wout.Middelhoven@wur.nl

\section{Systematics of the anamorphic basidiomycetous yeast genus Trichosporon Behrend with the description of five novel species: Trichosporon vadense, $T$. smithiae, $T$. dehoogii, $T$. scarabaeorum and T. gamsii}

\author{
Wouter J. Middelhoven, ${ }^{1}$ Gloria Scorzetti ${ }^{2}$ and Jack W. Fell ${ }^{2}$ \\ ${ }^{1}$ Laboratorium voor Microbiologie, Wageningen University, Hesselink van Suchtelenweg 4, \\ 6703 CT Wageningen, the Netherlands \\ ${ }^{2}$ Rosenstiel School of Marine and Atmospheric Sciences, University of Miami, \\ 4600 Rickenbacker Causeway, Key Biscayne, Florida 33149, USA
}

\section{INTRODUCTION}

Trichosporon Behrend is a genus of anamorphic yeasts (Basidiomycota, Hymenomycetes, Tremelloidae, Trichosporonales) with distinct morphological characteristics of budding yeast cells and true mycelia that disarticulate to form arthroconidia. The genus consists of soil- and waterassociated species, although some species are causative agents of diseases in man and cattle (Kwon-Chung \& Bennett, 1992; Guého et al., 1998; de Hoog et al., 2000).

Published online ahead of print on 21 November 2003 as DOI 10.1099/ijs.0.02859-0.

Abbreviations: IGS, intergenic spacer; ITS, internal transcribed spacer; LSU, large-subunit; SSU, small-subunit.

The GenBank/EMBL/DDBJ accession numbers for the ITS region sequences of strains CBS $8901^{\top}$, CBS $8370^{\top}$, CBS $8686^{\top}$, CBS $5601^{\top}$ and $\mathrm{CBS} 8245^{\top}$ are AY093425, AF444397, AF444476, AF444446 and AF444424, respectively. The accession numbers for their D1/D2 region sequences are AY093426, AF444706, AF444718, AF444710 and AF444708, respectively.

A phylogenetic tree based on sequencing of the D1/D2 region and a dichotomous identification key to 36 Trichosporon species are available as supplementary material in IJSEM Online.
With the exception of Trichosporon pullulans, the genus is monophyletic as viewed by small-subunit (SSU) rDNA (Guého et al., 1992; Sugita \& Nakase, 1998), D1/D2 region of the large-subunit (LSU) rDNA (Guého et al., 1998; Fell et al., 2000) and internal transcribed spacer (ITS) region (Sugita et al., 1999; Scorzetti et al., 2002) sequence analyses. Guého et al. (1998), in a review of the genus, accepted 19 species. Sugita \& Nakase (1998), based on SSU rDNA analysis of type strains, reported that the genus could be divided into three major groups of distinct species that can be distinguished by serotyping.

The standard technique for species identification of yeasts is based on phenotypic characteristics, including assimilation of about 50 carbon compounds and 10 nitrogen compounds. A major difficulty in obtaining accurate identification of basidiomycetous yeasts is the fact that different strains within a species respond differently to many of these tests. Consequently, strains can be misidentified and the existence of genetically distinct species can be overlooked. This difficulty can be overcome by nuclear base sequencing of rDNA.

Most of the physiological characteristics that are used 
in standard yeast descriptions depend on the activity of specific permeases, hydrolases or dehydrogenases. For most of the carbohydrates and polyalcohols tested, presence of these enzymes links these carbon compounds to central metabolism. In contrast, assimilation of uric acid and phenolic compounds requires the activity of more complex catabolic and anabolic pathways. As some of these compounds are found exclusively in specific environments, their assimilation may give information on the ecology of yeast species. These considerations inspired us to study growth responses to uric acid, ethylamine, polygalacturonate and some phenolic compounds for the distinction of Trichosporon species, as an alternative to traditional methods and base sequencing. Previous studies have demonstrated species-specific responses to these compounds (Middelhoven, 1993; Middelhoven et al., 1985, 1999, 2000, 2001; Sampaio, 1999). In some ascomycetous yeasts, growth on such compounds confirmed their phylogenetic relationships (Middelhoven \& Kurtzman, 2003). In the present study, we report growth responses to these compounds by strains of all currently accepted species of the genus Trichosporon. An identification key, based on these growth tests, assimilation of erythritol and L-rhamnose and maximum growth temperature, is available as supplementary material in IJSEM Online. The present study proposes five novel species in the genus Trichosporon, based on nuclear base sequencing and traditional and non-traditional growth tests.

\section{METHODS}

Source and characterization of strains. Strains studied are listed in Table 1. Strains of the novel species were examined for morphological and physiological properties by using standard yeast identification methods (Yarrow, 1998). Utilization of carbon sources $(0.5 \%)$ was tested in liquid Difco yeast nitrogen base (YNB) at $25^{\circ} \mathrm{C}$ on a rotary shaker at 100 r.p.m. The $\mathrm{pH}$ of growth media was adjusted to 5.5 as required; however, the $\mathrm{pH}$ of media with galacturonic acid or quinic acid was not adjusted, which is in agreement with the laboratory practice of CBS Delft (D. Yarrow, personal communication; Middelhoven, 1997). D-Glucaric (saccharic), L-malic, galactaric (mucic, $0 \cdot 25 \%$ ) and tartaric acids were tested at $\mathrm{pH} 4 \cdot 0$. Assimilation of nitrogen sources ( $40 \mathrm{mM}$ assimilable nitrogen) was tested in liquid yeast carbon base (YCB) except for sodium nitrite, which was tested by the auxanographic technique.

Basal growth medium (Middelhoven et al., 1991) that was used for non-traditional growth tests had the composition of YNB, but the phosphate concentration was tenfold higher in order to improve the buffering capacity. The nitrogen source, when present, was $2 \mathrm{~g}$ ammonium chloride $1^{-1}$, rather than $5 \mathrm{~g}$ ammonium sulphate $\mathrm{l}^{-1}$, as used in YNB. A droplet of a pre-culture in $2 \mathrm{ml}$ glucose $\left(5 \mathrm{~g} \mathrm{l}^{-1}\right)$ basal growth medium was inoculated into $2 \mathrm{ml}$ medium in culture tubes of $150 \times 15 \mathrm{~mm}$, which were then incubated on a rotary shaker at $25^{\circ} \mathrm{C}$. No ammonium chloride was added to growth media with nitrogenous substrates, such as uric acid, ethylamine, L-phenylalanine and L-4hydroxyproline. Uric acid ( $10 \mathrm{~g} \mathrm{l}^{-1}, 20 \mathrm{mg}$ per tube, $\mathrm{pH}$ not adjusted) was tested as the sole source of carbon and nitrogen. Tubes were agitated at $25^{\circ} \mathrm{C}$ to keep the crystals in suspension. Sparse but discernible growth at the expense of this energy-poor and weakly soluble substrate was assessed after disappearance of the crystals. Growth on the potentially toxic phenolic compounds 4-ethylphenol, orcinol, phloroglucinol, 2,3-dihydroxybenzoate and tyramine was studied in slant cultures (Middelhoven et al., 1991). Slants of basal growth medium (20 g agar $1^{-1}, 7 \mathrm{ml}$ per culture tube) were dried overnight and the agar surface was inoculated with the pre-culture. Substrates $(7 \mathrm{mg}$ in $0.1 \mathrm{ml})$ were added to the bottom of the slant, avoiding contact with the agar surface as much as possible. Substrates reached the cells by diffusion. Assimilation was deduced from a zone of growth higher or lower in the tube, depending on the substrate's toxicity.

Sequence analysis. ITS and D1/D2 rDNA molecular sequencing and analyses were performed at the University of Miami by using procedures described previously (Fell et al., 2000; Scorzetti et al., 2002). Phylogenetic analysis employed PAUP ${ }^{*} 4.0 \mathrm{~b} 10$ with maximumlikelihood analysis, a heuristic search with the starting tree obtained by neighbour-joining and tree bisection-reconnection. Gaps were handled as missing data. Due to time and computer constraints, bootstrap values were obtained with parsimony analysis. Bootstrap values were based on 1000 replications; values $<50 \%$ were not recorded. Complete D1/D2 and ITS region sequences are available in GenBank (Scorzetti et al., 2002). ITS region phylogenetic data are recorded in Fig. 1 and the D1/D2 analysis is available as supplementary material in IJSEM Online.

\section{RESULTS AND DISCUSSION}

\section{Latin diagnosis of Trichosporon vadense sp. nov. Middelhoven, Scorzetti et Fell}

In medio liquido dextrosum et peptonum et extractum levidinis et extractum malti continente post 3 dies ad $25^{\circ} \mathrm{C}$ hyphae septatae ramosae et pseudomycelium fragmentans formantur, neque cellulae zymosae gemmantes. Sedimentum et pellicula tenuis formantur, quae etiam post 4 hebdomades adsunt. Cultura in agaro PDA dicto post 3 dies cremea, butyrosa, non nitida, non elevata, mycelio fimbriata; post hebdomades $4,20^{\circ} \mathrm{C}$, eadem forma. In agaro YMA dicto post dies $3,20^{\circ} \mathrm{C}$, cellulae ovoideae et hyphae fragmentantes formantur. Fermentatio nulla. D-Glucosum, D-galactosum, acetyl-D-glucosaminum, D-ribosum, D-xylosum, L-arabinosum (lente), L-rhamnosum (lente), sucrosum, maltosum, trehalosum, methyl- $\alpha$-D-glucosidum, cellobiosum, salicinum (lente), arbutinum, melibiosum, lactosum, raffinosum (lente), glycerolum, ribitolum (lente), xylitolum (lente), D-glucitolum, D-mannitolum, inositolum, acidum gluconicum, acidum 2-ketogluconicum, acidum 5-ketogluconicum, acidum glucuronicum, acidum lacticum, acidum succinicum, acidum citricum (lente), ethanolum, propano-1,2-diolum, acidum galactonicum assimilantur, neque L-sorbosum, D-glucosaminum, melezitosum, inulinum, amylum solubile, erythritolum, L-arabinitolum, galactitolum, gluconolactonum, acidum galacturonicum, methanolum, butano-2,3-diolum, acidum quinicum. Aethylaminum, L-lysinum, cadaverinum et glucosaminum (lente) assimilantur, neque kalii nitratum, natrii nitritum, creatinum, creatininum, imidazolum et D-tryptophanum. Thiaminum externum crescentiae necessarium. Reactio Diazonii coerulaei B positiva. $30^{\circ} \mathrm{C}$ crescit neque $33^{\circ} \mathrm{C}$. Ureum finditur. Materia amyloidea formatur (lente).

Typus CBS $8901^{\mathrm{T}}$ isolatus ex terra prope Wageningen in Neerlandia, lyophilus praeservatus in collectione zymotica Centraalbureau voor Schimmelcultures, Utrecht, the Netherlands. 
Table 1. Strains studied

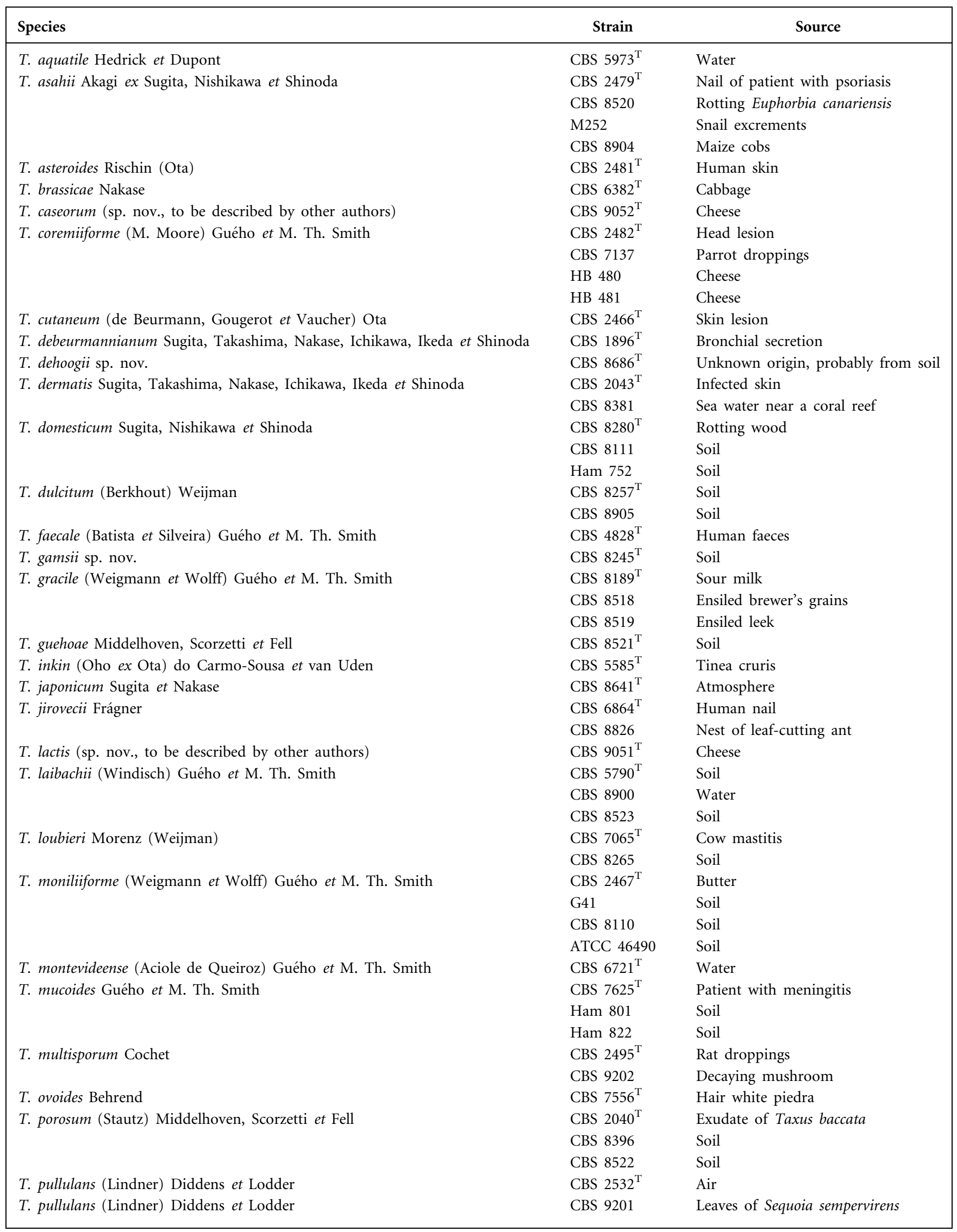


Table 1. cont.

\begin{tabular}{|c|c|c|}
\hline Species & Strain & Source \\
\hline T. scarabaeorum sp. nov. & CBS $5601^{\mathrm{T}}$ & Scarab beetle \\
\hline \multirow[t]{2}{*}{ T. smithiae sp. nov. } & CBS $8370^{\mathrm{T}}$ & Soil \\
\hline & CBS 9249 & Soil \\
\hline T. sporotrichoides (van Oorschot) van Oorschot et de Hoog & CBS $8246^{\mathrm{T}}$ & Soil \\
\hline T. vadense sp. nov. & CBS $8901^{\mathrm{T}}$ & Soil \\
\hline T. veenhuisii Middelhoven, Scorzetti et Fell & CBS $7136^{\mathrm{T}}$ & Buffalo dung \\
\hline Hyalodendron lignicola Diddens var. undulatum Diddens & CBS $220 \cdot 34^{\mathrm{T}}$ & Wood pulp \\
\hline Hyalodendron lignicola Diddens var. simplex Diddens & CBS $221 \cdot 34^{\mathrm{T}}$ & Wood pulp \\
\hline Novel species to be described elsewhere & CBS 8903 & Soil \\
\hline
\end{tabular}

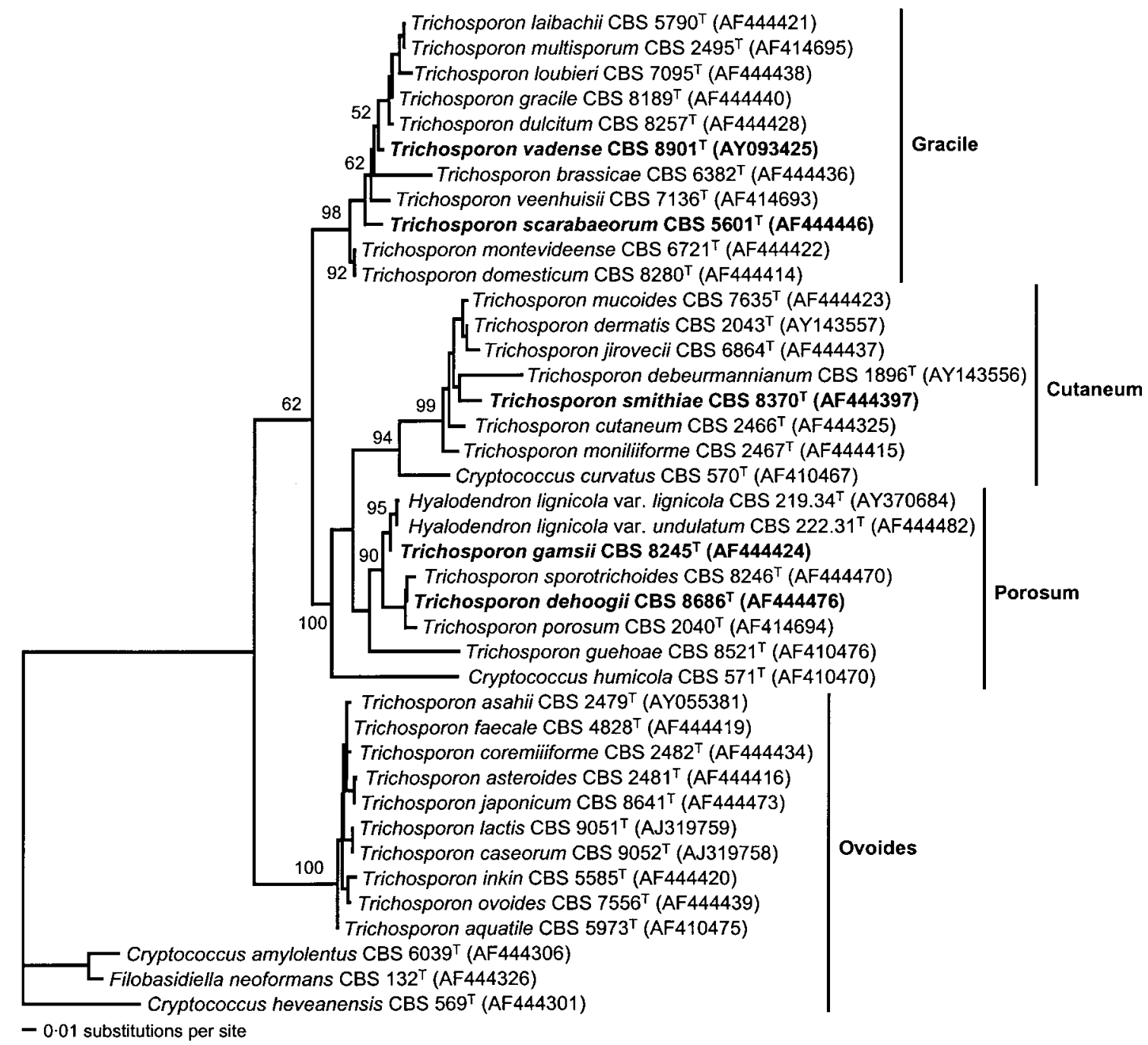

Fig. 1. Phylogenetic tree of the genus Trichosporon Behrend, based on nuclear sequencing of the ITS region of rDNA. Phylogenetic analysis (PAUP 4.0b10) was done by using maximum-likelihood and a heuristic search. Numbers on branches are bootstrap percentages ( $>50 \%$ ) from 1000 full heuristic replications, based on parsimony analysis. Cryptococcus amylolentus, Filobasidiella neoformans and Cryptococcus heveanensis were used as the outgroup. 


\section{Description of Trichosporon vadense sp. nov.}

Trichosporon vadense (va.den'se. L. neut. adj. vadense referring to the Latin name of Wageningen, Vila Vada; during the Roman age, Vila Vada was a small settlement and garrison on the northern bank of the river Rhine at the northern limit of the Roman empire on the European continent).

After 3 days at $25^{\circ} \mathrm{C}$ in liquid growth medium that contains glucose $(1 \%, \mathrm{w} / \mathrm{v})$, yeast extract $(0 \cdot 3 \%, \mathrm{w} / \mathrm{v})$, peptone $(0.5 \%, \mathrm{w} / \mathrm{v})$ and malt extract $(0.3 \% \mathrm{w} / \mathrm{v})$, abundant branched septate mycelium and fragmenting pseudomycelium, but no budding yeast cells, are formed. A sediment and a thin pellicle are formed, which remain present after 4 weeks. Slant cultures on YM agar after 3 days at $25^{\circ} \mathrm{C}$ have a butyrous texture and are cream-coloured and dull in appearance with entire to lobed margins; their appearance does not change over 4 weeks. In slide cultures on yeast morphology agar (YMA) after 3 days at $20^{\circ} \mathrm{C}$, masses of ovate cells budding on a broad base are formed. Under the cover slip, the mycelium splits into long fragments (five times as long as wide). Chains of ovate cells, budding on a broad base, are formed terminally on hyphae. Growth responses of strain CBS $8901^{\mathrm{T}}$ on standard carbon and nitrogen compounds, as well as some other characteristics, are shown in Table 2. The predominant ubiquinone is CoQ9.

The type strain $\left(\right.$ CBS $\left.8901^{\mathrm{T}}=\mathrm{JCM} 12194^{\mathrm{T}}\right)$ was isolated in 1990 by W. J. Middelhoven from soil in Wageningen, the Netherlands, by enrichment culture on phenylacetic acid at $10{ }^{\circ} \mathrm{C}$.

\section{Latin diagnosis of Trichosporon smithiae sp. nov. Middelhoven, Scorzetti, Sugita et Fell}

In medio liquido dextrosum et peptonum et extractum levidinis et extractum malti continente post 3 dies $25^{\circ} \mathrm{C}$ hyphae septatae ramosae formantur, cellulis globoseis ad ovoideis $(5-6.5 \times 6 \cdot 5-8.5 \mu \mathrm{m})$, singulis vel binis. Sedimentum parcum repens, et pellicula alba, rugosa repens formantur, quae etiam post 4 hebdomades adsunt. Cultura in agaro PDA dicto post 3 dies cremea, butyrosa, non nitida, elevata et mycelio fimbriata; post hebdomades $4,20^{\circ} \mathrm{C}$, eadem forma. In agaro YMA dicto post dies $3,20^{\circ} \mathrm{C}$, mycelium, pseudomycelium et arthroconidia formantur. Fermentatio nulla. DGlucosum, D-galactosum, acetyl-D-glucosaminum, D-ribosum, D-xylosum, L-arabinosum, L-rhamnosum, sucrosum, maltosum, trehalosum, methyl- $\alpha$-D-glucosidum, cellobiosum, arbutinum, melibiosum, lactosum, raffinosum, melezitosum, amylum solubile, glycerolum (lente), erythritolum, ribitolum (lente), xylitolum (lente), L-arabinitolum (lente), D-glucitolum (lente), D-mannitolum, inositolum, acidum gluconicum, acidum 2-ketogluconicum, acidum glucuronicum, acidum lacticum, acidum succinicum, acidum citricum, aethanolum, propano-1,2-diolum et acidum galactonicum (lente) assimilantur. L-Sorbosum, D-glucosaminum, D-arabinosum, salicinum, inulinum, galactitolum, gluconolactonum, acidum galacturonicum, methanolum, butano-2,3-diolum et acidum quinicum non assimilantur. Aethylaminum, L-lysinum, cadaverinum et glucosaminum (lente) assimilantur, neque kalii nitratum, natrii nitritum, creatinum, creatininum, imidazolum et D-tryptophanum. Thiaminum externum crescentiae necessarium. Reactio Diazonii coerulaei B positiva. $30^{\circ} \mathrm{C}$ crescit neque $32^{\circ} \mathrm{C}$. Ureum finditur. Materia amyloidea non formatur.

Typus CBS $8370^{\mathrm{T}}$ isolatus ex terra prope Angra dos Reis in Brasilio, lyophilus praeservatus in collectione zymotica Centraalbureau voor Schimmelcultures, Utrecht, the Netherlands.

\section{Description of Trichosporon smithiae sp. nov.}

Trichosporon smithiae [smi'thi.ae. N.L. gen. n. smithiae in honour of Maudy Th. Smith of CBS Delft/Utrecht, specialist in ascomycetous yeast systematics and co-author of revisions of the genus Trichosporon (Guého et al., 1992, 1998)].

After 3 days at $25^{\circ} \mathrm{C}$ in liquid growth medium that contains glucose $(1 \%, \mathrm{w} / \mathrm{v})$, yeast extract $(0 \cdot 3 \%, \mathrm{w} / \mathrm{v})$, peptone $(0 \cdot 5 \%, \mathrm{w} / \mathrm{v})$ and malt extract $(0 \cdot 3 \% \mathrm{w} / \mathrm{v})$, septate branched mycelium, budding pseudomycelium and budding globose to ovate cells $(5-6 \cdot 5 \times 6 \cdot 5-8.5 \mu \mathrm{m})$ are present. A flocculent sediment and a thick, wrinkled, white creeping pellicle are formed, which are present after 4 weeks. Slant cultures on PDA agar after 3 days at $25^{\circ} \mathrm{C}$ have a butyrous texture and are wrinkled, cream-coloured and dull in appearance with lobed margins. After 4 weeks, cultures are yellowish. In slide cultures on YMA after 3 days at $25^{\circ} \mathrm{C}$, abundant true mycelium with arthroconidia is formed. Growth responses of strain CBS $8370^{\mathrm{T}}$ on standard carbon and nitrogen compounds and other characteristics are shown in Table 2.

The type strain $\left(\right.$ CBS $8370^{\mathrm{T}}=$ DBVPG $\left.4506^{\mathrm{T}}=\mathrm{JCM} 12195^{\mathrm{T}}\right)$ was isolated in 1995 by Guido Capriotti from soil in Angra dos Reis, Brazil.

During the preparation of this manuscript, Dr T. Sugita brought a strain (M-9955) to our attention that he had isolated from soil in Japan. Its D1/D2 nucleotide sequence (GenBank accession no. AB086381) is identical to the sequence of CBS $8370^{\mathrm{T}}$. Strain M-9955 has been deposited in the CBS yeast culture collection (accession no. CBS 9249). Physiologically, CBS 9249 differs from the type strain by failure to grow on ribitol, xylitol and L-arabinitol, compounds that support weak and slow growth of CBS $8370^{\mathrm{T}}$.

\section{Latin diagnosis of Trichosporon dehoogii sp. nov. Middelhoven, Scorzetti et Fell}

In medio liquido dextrosum et peptonum et extractum levidinis et extractum malti continente post 3 dies $25^{\circ} \mathrm{C}$ cellulae globosae ad ovoideae (5-6.5 ×6.5-7.5 $\mu \mathrm{m})$, singulae vel binae, parcum pseudomycelium $(6 \cdot 5 \times 30 \mu \mathrm{m})$ formatur. Sedimentum densum album et pellicula tenuis repens formantur, quae etiam post 4 hebdomades adsunt. Cultura in agaro PDA dicto post 3 dies albida, butyrosa, non nitida, non elevata, mycelio fimbriata; post hebdomades $4,20^{\circ} \mathrm{C}$, 
Table 2. Physiological characteristics of the five novel Trichosporon species

Strains: 1, T. vadense CBS $8901^{\mathrm{T}}$; 2, T. smithiae CBS $8370^{\mathrm{T}}$; 3, T. dehoogii CBS $8686^{\mathrm{T}}$; 4, T. scarabaeorum CBS $5601^{\mathrm{T}}$; 5, T. gamsii CBS $8245^{\mathrm{T}}$. All five type strains grew on D-glucose, D-ribose, D-xylose, maltose, $\alpha$-trehalose, cellobiose, lactose, 2-ketogluconate, D-gluconate, D-glucuronate, DL-lactate, ethanol, Tween 80 and acetyl-D-glucosamine as sole carbon sources and on L-lysine and cadaverine as sole nitrogen sources. All five strains failed to grow on inulin, methanol, butane-2,3-diol, meso-tartrate and ethylene glycol as carbon sources and on nitrate, imidazole and D-tryptophan as nitrogen sources. All five strains required thiamin as the sole external vitamin for growth and showed positive responses to the urease reaction and the diazonium blue B stain. None of the strains grew in $10 \% \mathrm{NaCl}$ or $5 \% \mathrm{D}$ glucose or at $\mathrm{pH} 9 \cdot 5$. +, Growth within 8 days; -, no growth after 20 days; D, growth after 8 days or more; w, weak growth response; Th, thiamin required.

\begin{tabular}{|c|c|c|c|c|c|}
\hline Characteristic & 1 & 2 & 3 & 4 & 5 \\
\hline \multicolumn{6}{|l|}{ Carbon compounds: } \\
\hline D-Galactose & + & + & + & $\mathrm{W}$ & + \\
\hline L-Sorbose & - & - & $\mathrm{D}$ & $\mathrm{D}$ & + \\
\hline D-Glucosamine & - & - & $\mathrm{D}$ & $\mathrm{D}$ & DW \\
\hline D-Arabinose & $\mathrm{W}$ & - & - & - & - \\
\hline L-Rhamnose & $\mathrm{D}$ & + & + & - & + \\
\hline Sucrose & + & + & + & DW & + \\
\hline Methyl $\alpha$-glucoside & + & + & $\mathrm{D}$ & - & + \\
\hline Salicin & $\mathrm{D}$ & - & $\mathrm{D}$ & - & $\mathrm{D}$ \\
\hline Arbutin & + & + & + & - & + \\
\hline Melibiose & + & + & + & - & + \\
\hline Raffinose & $\mathrm{D}$ & + & + & - & - \\
\hline Melezitose & - & + & + & - & + \\
\hline Soluble starch & - & + & + & $\mathrm{D}$ & + \\
\hline Glycerol & + & $\mathrm{D}$ & $\mathrm{D}$ & + & $\mathrm{D}$ \\
\hline meso-Erythritol & - & + & + & - & - \\
\hline Ribitol & $\mathrm{D}$ & $\mathrm{D}$ & - & + & - \\
\hline Xylitol & $\mathrm{D}$ & $\mathrm{D}$ & - & DW & $\mathrm{D}$ \\
\hline L-Arabinitol & - & $\mathrm{D}$ & - & - & - \\
\hline D-Glucitol & + & $\mathrm{D}$ & $\mathrm{D}$ & $\mathrm{D}$ & + \\
\hline D-Mannitol & + & + & $\mathrm{D}$ & + & $\mathrm{D}$ \\
\hline Galactitol & - & - & + & - & - \\
\hline myo-Inositol & + & + & + & - & + \\
\hline Glucono- $\delta$-lactone & - & - & + & - & + \\
\hline 5-Ketogluconate & + & $\mathrm{D}$ & + & + & + \\
\hline Galacturonic acid & - & - & + & - & + \\
\hline Succinate & + & + & + & $\mathrm{D}$ & + \\
\hline Citrate & $\mathrm{D}$ & + & + & - & + \\
\hline Propane-1,2-diol & + & + & $\mathrm{D}$ & + & + \\
\hline Quinic acid & - & - & + & - & + \\
\hline Hemiglucarate & + & - & + & - & + \\
\hline Galactonate & + & $\mathrm{D}$ & + & + & + \\
\hline Palatinose & + & + & + & - & + \\
\hline Levulinate & + & - & $\mathrm{D}$ & - & - \\
\hline L-Malate & + & $\mathrm{D}$ & + & DW & + \\
\hline L-Tartrate & - & - & + & - & - \\
\hline D-Tartrate & - & - & + & - & - \\
\hline Galactarate & $\mathrm{D}$ & - & + & - & + \\
\hline Gentobiose & + & $\mathrm{D}$ & + & - & + \\
\hline Tween 60 & + & $\mathrm{D}$ & + & + & + \\
\hline \multicolumn{6}{|l|}{ Nitrogen compounds: } \\
\hline Nitrite & - & - & - & - & + \\
\hline Ethylamine (N) & + & + & + & $\mathrm{D}$ & + \\
\hline
\end{tabular}


Table 2. cont.

\begin{tabular}{|lccccc|}
\hline Characteristic & $\mathbf{1}$ & $\mathbf{2}$ & $\mathbf{3}$ & $\mathbf{4}$ & $\mathbf{5}$ \\
\hline Creatine & - & - & + & - & $\mathrm{D}$ \\
Creatinine & - & - & + & - & + \\
D-Glucosamine (N) & $\mathrm{D}$ & $\mathrm{DW}$ & $\mathrm{DW}$ & - & - \\
D-Proline & + & + & + & + & $\mathrm{D}$ \\
Putrescine & $\mathrm{D}$ & - & + & - & + \\
Urotropine & + & $\mathrm{DW}$ & + & $\mathrm{DW}$ & + \\
Miscellaneous: & & & & & - \\
Cycloheximide $(0 \cdot 01 \%)$ & + & + & + & + & - \\
Cycloheximide $(0 \cdot 1 \%)$ & $\mathrm{D}$ & $\mathrm{D}$ & - & $\mathrm{D}$ & + \\
Growth at pH 3.0 & $\mathrm{DW}$ & $\mathrm{DW}$ & + & - & $\mathrm{D}$ \\
Amyloid production & $\mathrm{D}$ & - & + & - & $27+, 29-$ \\
Maximum growth temp. $\left({ }^{\circ} \mathrm{C}\right)$ & $30+, 33-$ & $30+, 32-$ & $30+, 32-$ & $33+, 35-$ \\
\end{tabular}

eadem forma. In agaro extracto malti confecto post dies 3, $20{ }^{\circ} \mathrm{C}$, hyphae septatae fragmentantes, pseudomycelium et blastoconidia formantur. Fermentatio nulla. D-Glucosum, D-galactosum, L-sorbosum (lente), D-glucosaminum (lente), acetyl-D-glucosaminum, D-ribosum, D-xylosum, L-arabinosum, L-rhamnosum, sucrosum, maltosum, trehalosum, methyl- $\alpha$-Dglucosidum (lente), cellobiosum, salicinum (lente), arbutinum, melibiosum, lactosum, raffinosum, melezitosum, amylum solubile, glycerolum (lente), erythritolum, ribitolum (lente), D-glucitolum (lente), mannitolum (lente), galactitolum, inositolum, gluconolactonum, acidum gluconicum, acidum 2-ketogluconicum, acidum 5-ketogluconicum, acidum glucuronicum, acidum galacturonicum, acidum lacticum, acidum succinicum, acidum citricum, aethanolum, propano-1,2diolum (lente), acidum quinicum et acidum galactonicum assimilantur. D-Arabinosum, inulinum, ribitolum, xylitolum, L-arabinitolum, methanolum, butano-2,3-diolum non assimilantur. Aethylaminum, L-lysinum, cadaverinum, creatinum, creatininum et glucosaminum (lente) assimilantur, neque kalii nitratum, natrii nitritum, imidazolum, D-tryptophanum. Thiaminum externum crescentiae necessarium. Reactio Diazonii coerulaei B positiva. $30^{\circ} \mathrm{C}$ crescit neque $32^{\circ} \mathrm{C}$. Ureum finditur. Materia amyloidea formatur.

Typus CBS $8686^{\mathrm{T}}$, origine ignota, lyophilus praeservatus in collectione zymotica Centraalbureau voor Schimmelcultures, Utrecht, the Netherlands.

\section{Description of Trichosporon dehoogii sp. nov.}

Trichosporon dehoogii [de.hoo'gi.i. N.L. gen. n. dehoogii in honour of G. S. de Hoog of CBS Baarn/Utrecht, specialist in systematics of yeast-like fungi and co-author of revisions of the genus Trichosporon (Guého et al., 1992, 1998)].

After 3 days at $25^{\circ} \mathrm{C}$ in liquid growth medium that contains glucose $(1 \%$, w/v), yeast extract $(0.3 \%$, w/v), peptone $(0.5 \%, \mathrm{w} / \mathrm{v})$ and malt extract $(0.3 \%, \mathrm{w} / \mathrm{v})$, ovate budding cells and globose single cells $(5-6 \cdot 5 \times 6 \cdot 5-7 \cdot 5 \mu \mathrm{m})$ are present. Some pseudomycelium $(6.5 \times 30 \mu \mathrm{m})$ is present. A dense white sediment and a thin creeping pellicle are formed, which remain present after 4 weeks. Slant cultures on PDA agar after 3 days at $25^{\circ} \mathrm{C}$ have a butyrous texture and are flat, white to cream-coloured, have a dull appearance and a fringe of mycelium; their appearance does not change over 4 weeks. In slide cultures on malt extract agar after 3 days at $25^{\circ} \mathrm{C}$, abundant, true septate, long, fragmented and branched mycelium with globose blastoconidia is formed. Growth responses of strain CBS $8686^{\mathrm{T}}$ on standard carbon and nitrogen compounds and other characteristics are shown in Table 2. The predominant ubiquinone is CoQ9.

The origin of the type strain of $T$. dehoogii $\left(\mathrm{CBS} 8686^{\mathrm{T}}=\right.$ JCM $12196^{\mathrm{T}}$ ) is unknown.

\section{Latin diagnosis of Trichosporon scarabaeorum sp. nov. Middelhoven, Scorzetti et Fell}

In medio liquido dextrosum et peptonum et extractum levidinis et extractum malti continente post 3 dies $25{ }^{\circ} \mathrm{C}$ cellulae ovoideae ad cylindricae $(5-6 \cdot 5 \times 8 \cdot 5-12.5 \mu \mathrm{m})$, singulae vel binae. Sedimentum albidum et pellicula formantur, quae etiam post 4 hebdomades adsunt. Cultura in agaro PDA dicto post 3 dies albida, butyrosa, non nitida, non elevata, post hebdomades $4,20^{\circ} \mathrm{C}$, cremea, eadem forma. In agaro extracto malti confecto post dies $3,20^{\circ} \mathrm{C}$, mycelium, pseudomycelium et arthroconidia formantur. Fermentatio nulla. D-Glucosum, L-sorbosum (lente), D-glucosaminum (lente), acetyl-D-glucosaminum, D-ribosum, D-xylosum, maltosum, trehalosum, cellobiosum, lactosum, amylum solubile (lente), glycerolum, ribitolum, D-glucitolum (lente), D-mannitolum, acidum gluconicum, acidum 2-ketogluconicum, acidum 5-ketogluconicum, acidum glucuronicum, acidum lacticum, acidum succinicum (lente), aethanolum, propano1,2-diolum et acidum galactonicum assimilantur. L-Arabinosum, D-arabinosum, L-rhamnosum, methyl- $\alpha-\mathrm{D}-$ glucosidum, salicinum, arbutinum, melibiosum, raffinosum, melezitosum, inulinum, erythritolum, L-arabinitolum, galactitolum, inositolum, gluconolactonum, acidum galacturonicum, acidum citricum, methanolum, butano-2,3-diolum, acidum quinicum non assimilantur. Aethylaminum (lente), L-lysinum, cadaverinum assimilantur, neque kalii nitratum, 
natrii nitritum, creatinum, creatininum, glucosaminum, imidazolum, D-tryptophanum. Thiaminum externum crescentiae necessarium. Reactio Diazonii coerulaei B positiva. $33^{\circ} \mathrm{C}$ crescit neque $35^{\circ} \mathrm{C}$. Ureum finditur. Materia amyloidea non formatur.

Typus CBS $5601^{\mathrm{T}}$ isolatus ex Coleopteris scarabaeideis, lyophilus praeservatus in collectione zymotica Centraalbureau voor Schimmelcultures, Utrecht, the Netherlands.

\section{Description of Trichosporon scarabaeorum sp. nov.}

Trichosporon scarabaeorum (sca.ra.bae.o' rum. N.L. gen. n. scarabaeorum derived from Scarabaeum, the Latin name of the scarabaeid beetle, from which the type strain was isolated).

After 3 days at $25^{\circ} \mathrm{C}$ in liquid growth medium that contains glucose $(1 \%, \mathrm{w} / \mathrm{v})$, yeast extract $(0.3 \%, \mathrm{w} / \mathrm{v})$, peptone $(0.5 \%, \mathrm{w} / \mathrm{v})$ and malt extract $(0 \cdot 3 \% \mathrm{w} / \mathrm{v})$, cells are $5-6.5 \times 8 \cdot 5-12.5 \mu \mathrm{m}$ and occur singly or in pairs. Budding is on a broad base. Some branched mycelium is also formed. White sediment and a thin pellicle are formed. After 4 weeks, a white sediment and a thin, dry, dull, creeping pellicle are present. Slant cultures on PDA agar after 3 days at $25^{\circ} \mathrm{C}$ have a butyrous texture, are flat, white and dull in appearance and their margins are entire; their appearance does not change over 4 weeks. In slide cultures on malt extract agar after 3 days at $25^{\circ} \mathrm{C}$, abundant ovate cells and mycelium with arthroconidia are formed. Growth responses of strain CBS $5601^{\mathrm{T}}$ on standard carbon and nitrogen compounds and other characteristics are shown in Table 2. Strain CBS $5601^{\mathrm{T}}$ is notable for its inability to assimilate inositol. The predominant ubiquinone is CoQ9.

The type strain $\left(\right.$ CBS $\left.5601^{\mathrm{T}}=\mathrm{JCM} 12198^{\mathrm{T}}\right)$ was isolated by C. E. Malan in South Africa from the gut of larva of a scarabaeid beetle (Coleoptera, Scarabaeidae).

\section{Latin diagnosis of Trichosporon gamsii sp. nov. Middelhoven, Scorzetti, Sigler et Fell}

In medio liquido dextrosum et peptonum et extractum levidinis et extractum malti continente post 3 dies $20^{\circ} \mathrm{C}$ hyphae ramosae, septatae formantur, et cellulae globosae ad ovoideae $(5-7 \cdot 5 \times 6 \cdot 5-15 \mu \mathrm{m})$, singulae vel binae. Sedimentum copiosum album et pellicula repens formantur, quae etiam post 4 hebdomades adsunt. Cultura in agaro PDA dicto post 3 dies cremea, butyrosa, non nitida, elevata, mycelio fimbriata; post hebdomades $4,20^{\circ} \mathrm{C}$, eadem forma. In agaro YMA dicto post dies $3,20^{\circ} \mathrm{C}$, hyphae septatae fragmentantes et blastoconidia formantur. Fermentatio nulla. D-Glucosum, D-galactosum, L-sorbosum, D-glucosaminum (lente), acetyl-D-glucosaminum, D-ribosum, D-xylosum, L-arabinosum, L-rhamnosum, sucrosum, maltosum, trehalosum, methyl- $\alpha$-D-glucosidum, cellobiosum, salicinum (lente), arbutinum, melibiosum, lactosum, melezitosum, amylum solubile, glycerolum (lente), xylitolum (lente), D-glucitolum, D-mannitolum (lente), inositolum, gluconolactonum, acidum gluconicum, acidum 2-ketogluconicum, acidum 5-ketogluconicum, acidum glucuronicum, acidum galacturonicum, acidum lacticum, acidum succinicum, acidum citricum, aethanolum, propano-1,2-diolum, acidum quinicum assimilantur. D-Arabinosum, raffinosum, inulinum, erythritolum, ribitolum, L-arabinitolum, galactitolum, methanolum, butano2,3-diolum non assimilantur. Natrii nitritum, aethylaminum, L-lysinum, cadaverinum, creatinum (lente), creatininum assimilantur. Kalii nitratum, glucosaminum, imidazolum, D-tryptophanum non assimilantur. Thiaminum externum crescentiae necessarium. Reactio Diazonii coerulaei B positiva. $27^{\circ} \mathrm{C}$ crescit neque $29^{\circ} \mathrm{C}$. Ureum finditur. Materia amyloidea formatur (lente).

Typus CBS $8245^{\mathrm{T}}$ isolatus a W. Gams ex terra humosa madida radicibus interspersis, Sierra Nevada de Santa Maria in Colombia, lyophilus praeservatus in collectione zymotica Centraalbureau voor Schimmelcultures, Utrecht, the Netherlands.

\section{Description of Trichosporon gamsii sp. nov.}

Trichosporon gamsii (gam'si.i. N.L. gen. n. gamsii in honour of W. Gams of CBS Baarn/Utrecht, specialist in fungal systematics and botanical Latin, who isolated the strain).

After 3 days at $25^{\circ} \mathrm{C}$ in liquid growth medium that contains glucose $(1 \%, \mathrm{w} / \mathrm{v})$, yeast extract $(0.3 \%, \mathrm{w} / \mathrm{v})$, peptone $(0.5 \%, \mathrm{w} / \mathrm{v})$ and malt extract $(0.3 \% \mathrm{w} / \mathrm{v})$, cells are $5-7 \cdot 5 \times 6 \cdot 5-15 \mu \mathrm{m}$ in size. A thick, white sediment and a slightly creeping thin pellicle are formed. After 4 weeks, a heavy white sediment and a dry, creeping pellicle are present. Slant cultures on PDA agar after 3 days at $25^{\circ} \mathrm{C}$ are membranous, wrinkled, cream-coloured and dull in appearance with lobed margins; their appearance does not change over 4 weeks. In slide cultures on YMA after 3 days at $25^{\circ} \mathrm{C}$, abundant, branched and septate mycelium with ovate blastoconidia are formed. Under the cover slip, budding ovate cells and long, fragmented hyphae (ten times as long as wide) are formed. Growth responses of strain CBS $8245^{\mathrm{T}}$ on standard carbon and nitrogen compounds and other characteristics are shown in Table 2. The predominant ubiquinone is CoQ9.

The type strain $\left(\right.$ CBS $\left.8245^{\mathrm{T}}=\mathrm{JCM} 12197^{\mathrm{T}}\right)$ was isolated by W. Gams from a sample of moist humus around roots that was collected by T. van der Hammen in the Sierra Nevada de Santa Maria, Colombia. In previous papers (Guého et al., 1998; Middelhoven et al., 2001), CBS 8245 was erroneously considered to be the type strain of Trichosporon sporotrichoides.

\section{Non-conventional carbon sources}

Table 3 records growth responses to carbon compounds that are not traditionally used in yeast taxonomics. The pattern of carbon-compound assimilation was speciesspecific for the majority of species (Table 3 ). An identification key for clinically relevant Trichosporon species, based on some of these characteristics, was published recently (Middelhoven, 2003). A dichotomous identification key 
Table 3. Diagnostic growth responses of 36 species of the genus Trichosporon Behrend

Characters: 1, uric acid; 2, polygalacturonate; 3, ethylamine; 4, L-4-hydroxyproline; 5, quinate pH 5.5; 6, orcinol; 7, phloroglucinol; 8, 4-ethylphenol; 9, 2,3-dihydroxybenzoate; 10, tyramine; 11, L-phenylalanine; 12, erythritol; 13, L-rhamnose; 14, melibiose; 15, raffinose; 16 , melezitose; 17 , galacturonic acid; 18 , maximum growth temperature $\left({ }^{\circ} \mathrm{C}\right)$.

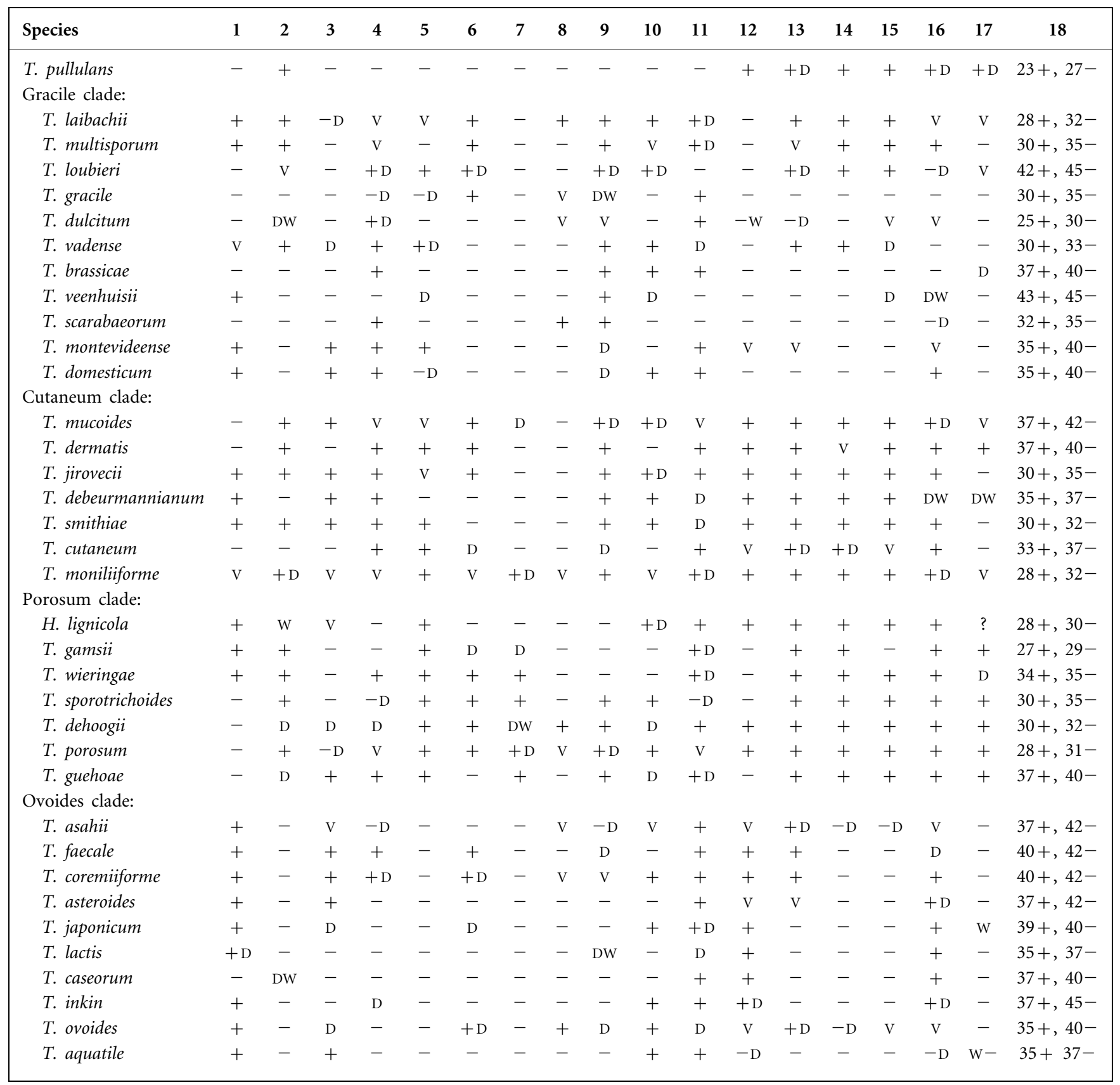

to 36 Trichosporon species, based on these growth tests, maximum growth temperature and assimilation of erythritol and L-rhamnose, is available as supplementary material in IJSEM Online. Delayed growth responses (D) are treated as + and - , so that strains can be identified after 1 week incubation. However, incubation for another week is recommended. Comparison of the data with those presented in Table 3 is required to confirm identification. Species that cannot be distinguished by standard techniques (e.g. Trichosporon asahii from Trichosporon coremiiforme and Trichosporon cutaneum from Trichosporon moniliiforme) can be separated by the following characters shown in Table 3: growth on L-4-hydroxyproline and orcinol, polygalacturonate, phloroglucinol and tyramine. In contrast, compounds to separate T. asahii and Trichosporon asteroides have not yet been found.

The benzene compounds listed in Table 4 are assimilated 
Table 4. Benzene compounds that are assimilated by almost all Trichosporon strains studied

Compounds are listed in order of the length of their side chain.

\begin{tabular}{|l|}
\hline Compound \\
\hline Phenol \\
Catechol (1,2-dihydroxybenzene) \\
Resorcinol (1,3-dihydroxybenzene) \\
Hydroquinone (1,4-dihydroxybenzene) \\
Benzoate \\
3-Hydroxybenzoate \\
4-Hydroxybenzoate \\
Protocatechuate (3,4-dihydroxybenzoate) \\
Phenylacetate \\
2-Hydroxyphenylacetate \\
3-Hydroxyphenylacetate \\
4-Hydroxyphenylacetate \\
Homoprotocatechuate (3,4-dihydroxyphenylacetate) \\
Cinnamate \\
3-Hydroxycinnamate \\
4-Hydroxycinnamate \\
Caffeate (3,4-dihydroxycinnamate)
\end{tabular}

as sole carbon sources by many yeast species, including species of Trichosporon (Middelhoven et al., 1992, 1999, 2000, 2001; Middelhoven, 1993; Sampaio, 1999). Consequently, these particular compounds have no diagnostic value, in contrast to other benzene compounds, e.g. orcinol, phloroglucinol, 4-ethylphenol, 2,3-dihydroxybenzoate, tyramine and L-phenylalanine, which can be used to distinguish species of Trichosporon (Table 3). T. pullulans is an exception: the type strain does not grow on any of these benzene compounds, thus confirming its remote phylogenetic relationship to other species of the genus.

Some compounds were included specifically in this study, due to their presence in natural habitats. Uric acid, which occurs in small amounts in soil, is the nitrogenous end product of protein catabolism in birds, reptiles and insects. Several yeast species utilize uric acid as the sole source of carbon and energy (Middelhoven et al., 1983, 1985, 1991). Some of these strains were associated with birds or had been isolated from soil by enrichment culture on uric acid. Growth on uric acid, which generally occurred within 5 days, proved to be a useful identification tool. All species of the genus Trichosporon with the exception of T. moniliiforme and $T$. vadense demonstrated definite positive or negative growth responses.

L-4-Hydroxyproline is a building block of collagen (which is found in cartilage, connective tissue and gelatin), from which the compound is released by enzymic hydrolysis. L-4-Hydroxyproline is assimilated by the pathogenic species $T$. coremiiforme, T. cutaneum, Trichosporon dermatis, Trichosporon inkin, Trichosporon jirovecii and Trichosporon loubieri, but not by Trichosporon asahii (some strains can assimilate it slowly), Trichosporon asteroides or
Trichosporon ovoides. Trichosporon mucoides showed variable growth responses to this hydroxyamino acid, which was also assimilated by some species that were unable to grow at $35^{\circ} \mathrm{C}$ (Trichosporon dulcitum, Trichosporon multisporum, T. scarabaeorum, T. smithiae and T. vadense).

Phloroglucinol and polygalacturonate are typical plant constituents. Few of the pathogenic species were able to assimilate these carbon compounds: phloroglucinol was assimilated by $T$. mucoides and polygalacturonate by T. dermatis, T. jirovecii and T. mucoides. T. loubieri was variable for this characteristic. Other typical plant constituents, such as quinic acid and orcinol, were assimilated by several pathogenic and non-pathogenic species.

\section{Phylogenetic analysis of the genus and novel species}

Sugita \& Nakase (1998) reported the presence of three groups of species within the genus Trichosporon, based on SSU rDNA analysis of 20 taxa. Our study (Fig. 1), with an expanded number of taxa $(n=36)$, concurs with the identity of these three groups, which correspond to our informal clade designations of Gracile (Sugita \& Nakase group III), Cutaneum (group I) and Ovoides (group II). Scorzetti et al. (2002) recognized a fourth clade, Hyalodendron, presently named Porosum, which was represented in the Sugita \& Nakase SSU rDNA tree as a separate branch. Several species within the Trichosporonales have not been included in our study: specifically, Trichosporon terricola (Sugita et al., 2002) became available too late for inclusion in the present study. The GenBank accession number for the sequence of the ITS and D1/D2 regions is AB086382. There are several Cryptococcus species among the Trichosporonales: Cryptococcus curvatus (Fig. 1 and Supplementary Figure, available in IJSEM Online) and Cryptococcus haglerorum (Middelhoven et al., 2003), which appear to be related to the Cutaneum clade, and Cryptococcus humicola, which may represent a separate cluster within or adjacent to the Trichosporonales (Fig. 1). Takashima et al. (2001) demonstrated that Cryptococcus musae, Cryptococcus ramirezgomezianus, Cryptococcus longus and Cryptococcus pseudolongus, which are phenotypically similar to C. humicola, represent a species complex within the Trichosporonales. The analysis by Takashima et al. (2001) was based on $18 \mathrm{~S}$ rDNA; sequences for the ITS and D1/D2 regions were not available for inclusion in our study. Those authors also reported Cryptococcus daszewskae to be related to C. curvatus. Clearly, a systematic evaluation of Cryptococcus species within the Trichosporonales is required.

Comparison of the ITS and D1/D2 Trichosporonales trees (Fig. 1 and Supplementary Figure, available in IJSEM Online) demonstrates more variability in the D1/D2 region than in the ITS region. For example, we observed no differences in the ITS sequence alignments between Trichosporon laibachii and T. multisporum, and seven differences (transitions : transversions, $6: 1$ ) in the D1/D2 region. 
Trichosporon montevideense and Trichosporon domesticum had identical sequences in the ITS region and differed at two positions (ti:tv, 1:1) in D1/D2. SSU rDNA analyses also demonstrated nucleotide position differences within these pairs of species (Sugita \& Nakase, 1998). The general assumption is that LSU rDNA is less variable than the ITS region. The reason for the lack of conformity to this assumption among the Trichosporonales is not clear. Yang \& Yoder (1999) examined ti : tv rate ratios and found that they varied between genes and lineages. These authors, however, could not find a biological explanation for these rate variations.

Previous studies (Middelhoven et al., 1999, 2000, 2001) that focused on the D1/D2 region provided ample sequence differences between species. However, the inclusion of the ITS region suggests that there may be closer phylogenetic relationships among species than was considered previously. In some cases, this relationship is reflected in phenotypic characteristics. A prime example is the species group T. coremiiforme, T. asteroides and T. asahii, which are similar in phenotypic characters and the ITS region, but differ in D1/D2 sequence. Species differentiation for such closely related species may require confirmation by using other sequences, such as rDNA intergenic spacer (IGS) sequences, as demonstrated by Sugita et al. (2002). These authors examined 43 strains of 25 species of Trichosporon in the IGS region and reported geographical substructure among strains of T. asahii and genetic differences between T. asahii, T. coremiiforme, Trichosporon faecale, T. asteroides and T. japonicum.

The five novel species, which reside in the Gracile, Porosum and Cutaneum clades, can be differentiated readily by genetic and phenotypic differences. The latter are shown in Tables 2 and 3. Distinction of the four clades by these phenotypic characters is difficult, confirming the monophyletic nature of the genus.

The Gracile clade has strong support in the ITS region (bootstrap value, 99\%). This clade includes the two novel species T. vadense and T. scarabaeorum. The Gracile clade is physiologically heterogeneous with respect to maximum growth temperature and assimilation of polygalacturonate, uric acid, orcinol, L-4-hydroxyproline, tyramine and Lphenylalanine. Most species do not grow on ethylamine, but $T$. domesticum and $T$. montevideense, which take a separate position in this clade, grow well on this amine as the sole source of carbon and nitrogen (Table 3). According to the ITS-based phylogenetic tree (Fig. 1), the closest relatives of $T$. vadense are $T$. laibachii, T. multisporum, T. loubieri, T. dulcitum, Trichosporon gracile and Trichosporon veenhuisii, although statistical support is weak $(52 \%)$. All of these species have some characteristics in common, e.g. failure to grow on erythritol, galacturonic acid (T. laibachii is variable) and phloroglucinol, no or delayed growth on ethylamine as the sole source of carbon and nitrogen and a maximum growth temperature of $35^{\circ} \mathrm{C}$ or less, with the exception of $T$. veenhuisii and
T. loubieri, which grow at $42{ }^{\circ} \mathrm{C}$. Physiologically, T. vadense is similar to T. laibachii and T. multisporum. These three species can be separated from each other by growth responses to melezitose, orcinol and 4-ethylphenol. $T$. vadense, T. laibachii and T. multisporum grow readily on polygalacturonate, but most strains do not grow on the monomer galacturonic acid. Growth tests on the latter are traditionally carried out without prior $\mathrm{pH}$ adjustment, resulting in an initial $\mathrm{pH}$ below 3.0 (Middelhoven, 1997). Assimilation of the polymer and failure to grow on the monomer reflect sensitivity to acid growth conditions.

T. scarabaeorum is not related closely to any species in the clade, although a possible relationship to Trichosporon brassicae, $T$ veenhuisii, T. montevideense and $T$. domesticum is depicted in the ITS-based tree (Fig. 1). All of these species have a maximum growth temperature between 35 and $40^{\circ} \mathrm{C}$, but that of $T$. scarabaeorum is between 32 and $35^{\circ} \mathrm{C}$. The novel species can be distinguished in the clade by a combination of responses to 4-ethylphenol and L-phenylalanine. Distinction of $T$. domesticum and $T$. montevideense within the clade can be achieved by their differential abilities to utilize quinate and tyramine. Separation by traditional growth tests is difficult, as many growth responses are variable.

The Porosum clade, which has $90 \%$ bootstrap support in the ITS tree, includes two novel species, T. gamsii and T. dehoogii, and the species Hyalodendron lignicola, T. sporotrichoides and Trichosporon porosum. The ITS tree suggests weak relationships of Trichosporon guehoae and C. humicola to these species, observations that are not supported by D1/D2 analysis (see Supplementary Figure in IJSEM Online). The Porosum clade is characterized by a low maximum growth temperature $\left(<37^{\circ} \mathrm{C}\right)$ and by growth on L-rhamnose, melibiose, raffinose (except T. gamsii), galacturonic acid and quinate. The novel species T. gamsii can be distinguished in the clade by the combined characters of assimilation of uric acid and failure to grow on raffinose and 2,3-dihydroxybenzoate. The other novel species in the clade, $T$. dehoogii, is similar to T. porosum, but does not assimilate L-arabinitol (Table 2) and utilizes creatine and creatinine as sole nitrogen sources. T. sporotrichoides can be distinguished from T. porosum and $T$. dehoogii by failure to grow on erythritol. The other member of the clade, $H$. lignicola, is characterized by very few or no budding yeast cells (de Hoog \& Smith, 1998).

The Cutaneum clade (without C. curvatus) is supported strongly by bootstrap analysis (Fig. 1) and is characterized by coenzyme Q10 as the main ubiquinone (Sugita \& Nakase, 1998) and by growth on polygalacturonate (except for Trichosporon debeurmannianum and T. cutaneum) and on 2,3-dihydroxybenzoate, erythritol, L-rhamnose and raffinose, with the exception of some strains of $T$. cutaneum. The Cutaneum clade is heterogeneous with respect to maximum growth temperature and assimilation of uric acid. The novel species $T$. smithiae does not appear to be related closely to any particular species in either tree. 
Distinction from other species in the clade can be based on growth responses to uric acid, polygalacturonate, orcinol and phloroglucinol.

The Ovoides clade is supported strongly by bootstrap analysis (100\%; Fig. 1). All species have a maximum growth temperature of $35-40{ }^{\circ} \mathrm{C}$, assimilate uric acid (except for Trichosporon caseorum) and L-phenylalanine (some strains are slow) and do not assimilate quinate or phloroglucinol. In addition, responses to melibiose, raffinose, galacturonic acid and polygalacturonate are negative for most strains.

\section{ACKNOWLEDGEMENTS}

Thanks are due to W. Gams, Utrecht, the Netherlands, for correcting the Latin diagnoses, to Elke Hammer, Greifswald, Germany, for determining the predominant coenzyme $\mathrm{Q}$ and for strains $T$. domesticum Ham 752 and T. mucoides Ham 801 and 822, to H.-J. Prillinger, Vienna, Austria, for strains T. coremiiforme HB 480 and HB 481 and for the type strains of $T$. caseorum and T. lactis prior to their publication, to Ann Vaughan-Martini for strain CBS 8370, to T. Sugita for strain CBS 9249 and to Lynne Sigler for strains and discussions regarding the phylogenetic placement of T. sporotrichoides and T. gamsii. Nucleotide sequencing and sequence analysis by J.W.F. and G. S. were supported by the USA National Science Foundation, Division of Environmental Biology (DEB 0206521).

\section{REFERENCES}

de Hoog, G. S. \& Smith, M. Th. (1998). Hyalodendron Diddens. In The Yeasts, a Taxonomic Study, 4th edn, pp. 773-774. Edited by C. P. Kurtzman \& J. W. Fell. Amsterdam: Elsevier.

de Hoog, G. S., Guarro, J., Gené, J. \& Figueras, M. J. (editors) (2000). Atlas of Clinical Fungi, pp. 164-175. Utrecht: Centraalbureau voor Schimmelcultures.

Fell, J. W., Boekhout, T., Fonseca, A., Scorzetti, G. \& StatzellTallman, A. (2000). Biodiversity and systematics of basidiomycetous yeasts as determined by large-subunit rDNA D1/D2 domain sequence analysis. Int J Syst Evol Microbiol 50, 1351-1371.

Guého, E., Smith, M. Th., de Hoog, G. S., Billon-Grand, G., Christen, R. \& Batenburg-van der Vegte, W. H. (1992). Contributions to a revision of the genus Trichosporon. Antonie van Leeuwenhoek 61, 289-316.

Guého, E., Smith, M. Th. \& de Hoog, G. S. (1998). Trichosporon Behrend. In The Yeasts, a Taxonomic Study, 4th edn, pp. 854-872. Edited by C. P. Kurtzman \& J. W. Fell. Amsterdam: Elsevier.

Kwon-Chung, K. J. \& Bennett, J. E. (1992). Medical Mycology. Philadelphia, PA: Lea \& Febiger.

Middelhoven, W. J. (1993). Catabolism of benzene compounds by ascomycetous and basidiomycetous yeasts and yeastlike fungi. A literature review and an experimental approach. Antonie van Leeuwenhoek 63, 125-144.

Middelhoven, W. J. (1997). Assimilation of organic acids: the $\mathrm{pH}$ as determining factor. Yeast 46, 19-20.

Middelhoven, W. J. (2003). Identification of clinically relevant Trichosporon species. Mycoses 46, 7-11.

Middelhoven, W. J. \& Kurtzman, C. P. (2003). Relation between phylogeny and physiology in some ascomycetous yeasts. Antonie van Leeuwenhoek 83, 69-74.
Middelhoven, W. J., van den Brink, J. A. \& Veenhuis, M. (1983). Growth of Candida famata and Trichosporon cutaneum on uric acid as the sole source of carbon and energy, a hitherto unknown property of yeasts. Antonie van Leeuwenhoek 49, 361-368.

Middelhoven, W. J., de Kievit, H. \& Biesbroek, A. L. (1985). Yeast species utilizing uric acid, adenine, n-alkylamines or diamines as sole source of carbon and energy. Antonie van Leeuwenhoek 51, 289-301.

Middelhoven, W. J., de Jong, I. M. \& de Winter, M. (1991). Arxula adeninivorans, a yeast assimilating many nitrogenous and aromatic compounds. Antonie van Leeuwenhoek 59, 129-137.

Middelhoven, W. J., Koorevaar, M. \& Schuur, G. (1992). Degradation of benzene compounds by yeasts in acidic soils. Plant Soil 145, 37-43.

Middelhoven, W. J., Scorzetti, G. \& Fell, J. W. (1999). Trichosporon guehoae sp. nov., an anamorphic basidiomycetous yeast. Can J Microbiol 45, 686-690.

Middelhoven, W. J., Scorzetti, G. \& Fell, J. W. (2000). Trichosporon veenhuisii sp. nov., an alkane-assimilating anamorphic basidiomycetous yeast. Int J Syst Evol Microbiol 50, 381-387.

Middelhoven, W. J., Scorzetti, G. \& Fell, J. W. (2001). Trichosporon porosum comb. nov., an anamorphic basidiomycetous yeast inhabiting soil, related to the loubieri/laibachii group of species that assimilate hemicelluloses and phenolic compounds. FEMS Yeast Res 1, 15-22.

Middelhoven, W. J., Fonseca, A., Carreiro, S. C., Pagnocca, F. C. \& Bueno, O. C. (2003). Cryptococcus haglerorum, sp. nov., an anamorphic basidiomycetous yeast isolated from nests of the leafcutting ant Atta sexdens. Antonie van Leeuwenhoek 83, 167-174.

Sampaio, J. P. (1999). Utilization of low molecular weight aromatic compounds by heterobasidiomycetous yeasts: taxonomic implications. Can J Microbiol 45, 491-512.

Scorzetti, G., Fell, J. W., Fonseca, A. \& Statzell-Tallman, A. (2002). Systematics of basidiomycetous yeasts: a comparison of large subunit D1/D2 and internal transcribed spacer rDNA regions. FEMS Yeast Res 2, 495-517.

Sugita, T. \& Nakase, T. (1998). Molecular phylogenetic study of the basidiomycetous anamorphic yeast genus Trichosporon and related taxa based on small subunit ribosomal DNA sequences. Mycoscience 39, 7-13.

Sugita, T., Nishikawa, A., Ikeda, R. \& Shinoda, T. (1999). Identification of medically relevant Trichosporon species based on sequences of internal transcribed spacer regions and construction of a database for Trichosporon identification. J Clin Microbiol 37, 1985-1993.

Sugita, T., Takashima, M., Nakase, T., Ichikawa, T., Shinoda, T. \& Nishikawa, A. (2002). A basidiomycetous anamorphic yeast, Trichosporon terricola sp. nov., isolated from soil. J Gen Appl Microbiol 48, 293-297.

Takashima, M., Sugita, T., Shinoda, T. \& Nakase, T. (2001). Reclassification of the Cryptococcus humicola complex. Int J Syst Evol Microbiol 51, 2199-2210.

Yang, Z. \& Yoder, A. D. (1999). Estimation of the transition/ transversion rate bias and species sampling. $J$ Mol Evol 48, 274-283.

Yarrow, D. (1998). Methods for the isolation, maintenance and identification of yeasts. In The Yeasts, a Taxonomic Study, 4th edn, pp. 77-100. Edited by C. P. Kurtzman \& J. W. Fell. Amsterdam: Elsevier. 